\title{
UKRN Position on Research Outputs
}

The UK Reproducibility Network Steering Group $29^{\text {th }}$ June 2021

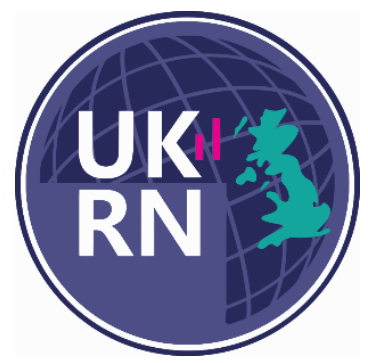

There is growing awareness of the value of making research artefacts - from data (broadly conceived) to final outputs (such as journal articles) - as open and available as they can be. Various benefits to this have been proposed, including improved accessibility to research users, greater scope for re-use, the potential for the research community to scrutinise entire workflows, and the opportunity for a wider range of research contributions to be recognised. A growing number of platforms - from institutional repositories to preprint servers - support the deposition of research artefacts.

Funders and publishers are increasingly adopting policies that encourage research transparency, including data sharing and open access publication. However, in our view the primary responsibility for making research artefacts as open and available as possible lies with the originators. The Rights Retention Strategy ${ }^{1}$ developed by cOAlition S enables researchers to fulfil this responsibility, by ensuring that if a CC BY license is not applied by the journal to the Version of Record (VoR; the final copyedited, typeset, and formatted version that is published), the authors assert this for their Author Accepted Manuscript (AAM; the final version submitted by the authors and accepted by the journal following changes made during peer review).

One concern is that these final research outputs (the AAM and the VoR) bring together multiple intellectual contributions including some - like the input of peer reviewers - external to the originating research team. Recognition of these contributions is sometimes possible - for example through open peer review, where the peer review record is published alongside the VoR. However, this remains the exception, and such contributions are typically not visible in the AAM. Linkage of research artefacts, including AAMs, with associated external intellectual contributions (such as peer review, where this is open) could further enhance transparency, if the originators of these external contributions are willing to be acknowledged. ORCID identifiers could help achieve this.

Making the research process transparent and accessible is more possible now than it has ever been, and it is increasingly encouraged (and even mandated) by funders, publishers and institutions. This brings challenges - in particular, the need for streamlined processes that allow this transparency to be achieved efficiently. Increasing the availability of AAMs through a CC BY licence is an innovative and practical approach to securing open access to final research outputs, but it is also important that intermediate research artefacts are made available, and contributions of the wider research community - such as peer reviewers acknowledged, where possible. Where the journal VoR includes such information, we encourage authors to update their AAM accordingly to reflect this.

${ }^{1}$ https://www.coalition-s.org/rights-retention-strategy

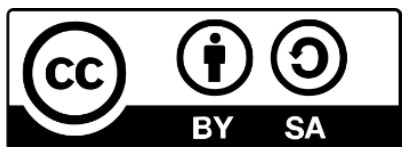

This work is licensed under a Creative Commons Attribution-ShareAlike 4.0 International License. 